\title{
A Review and Analysis of the Literature on Public Health Emergency Communication Practices
}

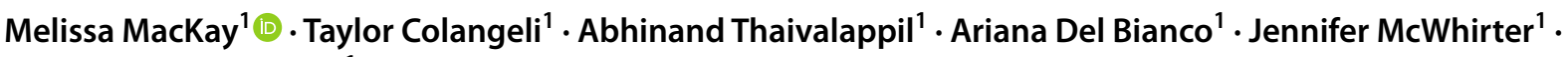 \\ Andrew Papadopoulos ${ }^{1}$
}

Accepted: 30 August 2021 / Published online: 13 September 2021

(c) The Author(s), under exclusive licence to Springer Science+Business Media, LLC, part of Springer Nature 2021

\begin{abstract}
A systematic review using structured and transparent methods was carried out to collect and review the qualitative literature investigating trust in crisis communication during emerging infectious diseases. Qualitative synthesis was conducted using a descriptive thematic analysis approach. The GRADE-CERQual assessment was used to determine the confidence in each thematic finding to support decisions when implementing review findings. Overall, 13 studies were included in the review, resulting in 10 thematic categories that describe characteristics associated with crisis communication information and sources of crisis communication that can enhance or maintain public trust. The results of this review suggest the public judges the trustworthiness of crisis communication based on the information characteristics, including consistency, repetition, and timeliness, and especially transparency and uncertainty. Public health is a trusted source of crisis communication when the presenting spokesperson is a health official, the information is not perceived as politicized, and is timely. Community leaders, such as family doctors, are also trusted sources of crisis communication, whereas media and government officials face distrust because of perceived sensationalized information, and defensiveness and unreliable information respectively. Qualitative data in this area is limited, especially involving the public and priority populations, and should be the focus of future research.
\end{abstract}

Keywords Trust $\cdot$ Emerging infectious disease $\cdot$ Qualitative systematic review $\cdot$ Crisis communication $\cdot$ Risk communication

\section{Introduction}

The importance of communication has been highlighted by the coronavirus disease of 2019 (COVID-19) pandemic, where building and maintaining public trust through crisis communication to promote recommended prevention behaviors (e.g., physical distancing) is essential to decrease virus transmission. By May 2020, the novel coronavirus spread to 188 countries and caused widespread travel bans and restrictions, along with measures to slow its spread [1]. As of July 2021, Canada has had almost 1.5 million cases and close to 27,000 deaths with multiple variants of concern circulating [1].

The uncertainty surrounding an emerging infectious disease coupled with the rapidly changing science create

Melissa MacKay

melissam@uoguelph.ca

1 Department of Population Medicine, University of Guelph, 50 Stone Road East, Guelph, ON N1G 2W1, Canada unique communication challenges [2]. Effective communication is necessary during pandemics to enable individuals to understand the evolving situation, maintain public trust and organizational credibility, and facilitate informed decisionmaking [3-8]. As Dr. Rishi Manchanda [9] stated "public health moves at the speed of trust", however, public health is contending against the spread of mis- and dis-information and a growing distrust of evidence, government, and experts [10]. A fundamental goal of crisis response is to ensure trust and credibility of the actors is positively maintained [11], which reduces the impact of pandemics through affecting the adopting of public health measures [5, 7, 11, 12].

Declining trust in science, experts, government, and healthcare has been reported in North America [10, 13]. Even a small drop in public trust can result in additional barriers to successful pandemic crisis communication [10]. Inconsistent or conflicting messaging and the politicization of COVID-related discourse has likely led to declining trust [14], and reduced adherence to COVID-19 prevention behaviours in Canada [15]. 
Qualitative studies allow for the in-depth exploration of multiple perspectives and experiences to understand factors associated with maintaining trust through crisis communication during pandemics. Our research aim was to conduct a systematic review and descriptive thematic analysis of previously published qualitative literature exploring factors related to effective crisis communication by public health and other actors to build and maintain public trust during emerging infectious diseases (EID) in high-income countries. No previous studies have conducted similar synthesis methods using thematic analysis to explore this topic. The results of this paper can help inform current and future crisis communication practices surrounding COVID-19 and other public health emergencies to maintain public trust while ensuring safe behaviors. Although distinct, risk and crisis communication are often used interchangeably in the literature, however, this research will refer to it as crisis communication to reflect the stage of the crisis unless it is a direct quote from the literature using risk.

\section{Methods}

Two independent reviewers contributed to the title and abstract screening, full text screening, data extraction, article quality appraisal, and the GRADE-Confidence in Findings from Qualitative Evidence Syntheses (CERQual). The Enhancing transparency in reporting the synthesis of qualitative research (ENTREQ) guideline was followed to allow for transparent reporting and interpretation of the review results [16].

\section{Search Strategy}

The search strategy contained concepts related to health, risk, and crisis communication, trust, and emerging infectious disease; it was tested and refined in Ovid MEDLINE (see Supplementary Fig. 1). A combination of Medical Subject Headings, controlled vocabulary, and keywords were used to identify peer-reviewed articles and grey literature in the following databases: Ovid MEDLINE, CAB Direct, PsycINFO, and Web of Science. Six peer-reviewed journals most cited by the largest number of relevant results from the database searches were hand-searched for articles published between 2009 to June 2020: Journal of Health Communication, Health Communication, Journal of Public Health, Journal of Infectious Diseases, Health Education and Behavior, and Emerging Infectious Diseases. Grey literature was captured through Google searches using simple search strings representing the concepts from the database searches (keyword examples: "trust + crisis communication + emerging infectious disease").

\section{Study Selection and Data Extraction}

All retrieved citations were imported into the review software DistillerSR (Evidence Partners, Ottawa, Canada) and duplicate articles were removed. Title and abstracts were screened using a structured screening form for relevance to the research question. The reviewers discussed conflicts and included studies for full text screening if they were unsure. Next, article full texts were screened based on the inclusion criteria using a structured form to capture relevant study characteristics. Kappa inter-rater reliability for this stage based on inclusion for the final review was 0.86 , indicating very high agreement between reviewers [17]. Reviewers again discussed and resolved any conflicts at this stage.

The following criteria were extracted from each full text article: title, authors, year of publication, publication type, country of origin, study design, study objective(s)/ research question(s), study population/sample size, sector/ context, aims/purpose, methodology, theories/frameworks, outcome characteristics of quantitative measures, biases identified, key findings, gaps/limitations, and future areas of study.

\section{Study Inclusion Criteria}

Studies conducted between 2009 and the time of the database search, June 2020, were included to capture research examining pandemics and outbreaks starting with with SARS, the first pandemic of the twenty-first century, which overlaps with the adoption of mainstream use of social media in the mid-2000s [18]. Original qualitative data from qualitative or mixed methods research published in English were included. The study population was based on high-income countries [19] where results were generalizable to the community, country, or global level. This review focused on high income countries to understand how trust is developed and maintained in countries with similar healthcare infrastructures, governing, and standards of living. Studies with EID at the outbreak, endemic, or pandemic scales that focused on health or risk or crisis communication and public trust or credibility were included. Studies were excluded if they focused on vector-borne and foodborne diseases; sexually transmitted infections and HIV/AIDS; chronic disease; bioterrorism; and vaccination only. Vaccination-only focused studies were excluded as although trust is a vital element to adoption, some of the best practices surrounding communicating vaccination differ from those of crisis communication. 


\section{Quality Appraisal}

Relevant articles underwent a quality assessment using a form developed by Thaivalappil et al. [20], adapted from a pre-existing quality assessment tool [21]. Key characteristics regarding study scope and purpose, study design, sampling strategy, analytic approach, study findings, researcher reflexivity, ethical issues, and study relevance and transferability were examined to determine the study quality [22]. The quality assessment was conducted by two researchers independently and discussed any conflicts to resolution.

\section{Analysis}

Thomas and Harden's [23] guidelines for thematic synthesis was followed to analyze and synthesize results from the included articles. Relevant data from the results, discussion, and conclusion sections were coded line-by-line into initial codes using an inductive approach and were periodically discussed to ensure agreement [23]. Descriptive themes were created after generating initial themes from the coded extracts and the data set, as well as creating a thematic map of analysis [23]. Two researchers independently participated in the ongoing analysis to refine themes until agreement was reached.

Three researchers independently completed the CERQual assessment for each theme and came together to develop a consensus for each criterion and overall rating. The confidence in each major theme was assessed based on the four criteria found in GRADE-CERQual; methodological limitations; relevance to the context of the review aim; coherence of findings related to data from primary studies and explanation of patterns found; and adequacy of the data in terms of quantity and sufficient detail [24]. Ratings for each criterion were ranked based on the level of concerns: no, minor, moderate, or substantial [24]. Based on the assessment of each of the four criteria for the thematic areas, an overall confidence was determined: high, moderate, low, and very low, which described the likelihood that the review finding was a reasonable representation of the phenomenon of interest [24]. The approach aims to transparently communicate how closely linked the review findings are to the focus of the study [25]. If findings are substantially different from the phenomenon of interest, the implementation in policy, practice, or programs may not be as described in the review finding [25].

\section{Synthesis}

\section{Study Characteristics}

Thirteen studies were included in the review from the original 6848 citations (Fig. 1) and a summary of their descriptive characteristics are presented in Table 1. Studies originated from eight high-income countries, with most conducted in Canada $(n=5)$. Study context varied in terms of infectious disease focus; $\mathrm{H} 1 \mathrm{~N} 1$ pandemic $(\mathrm{n}=5)$; COVID-19 $(\mathrm{n}=2)$; SARS $(\mathrm{n}=1)$; Ebola $(\mathrm{n}=1)$; fictitious EID $(n=1)$; and unspecified EID $(n=3)$. Most studies were journal articles $(n=12)$ with one dissertation included. Although grey literature was searched and screened for, it did not meet the study inclusion criteria and was not included in this review. Supplementary Table 1 presents a summary describing the design, country of origin, context, and key findings of the included studies.

\section{Quality Appraisal Results}

A summary of quality appraisal results is found in Table 1, and full results are available in Supplementary Table 2. The main findings included a lack of researcher reflexivity reported $(n=9)$ and a lack of complete description of study relevance or transferability $(n=7)$, which lacked inclusion of future directions, limitations, or bias in the discussion. No studies were excluded from this review based on the quality appraisal.

\section{Thematic Analysis}

Ten descriptive themes were classified and described. Table 2 describes the overall confidence for each theme based on the CERQual approach, and Supplementary Table 2 contains a breakdown of the CERQual ratings for each theme.

A conceptual framework [Fig. 2] illustrates the relationship of essential factors for effective communication that impact trustworthiness and trust in crisis communication. The conceptual framework identifies significant relationships, found in the included literature, between the characteristics of crisis communication, communication channels, the sources that provide it, and ultimately trust.

\section{Consistency of the Information}

Moderate confidence was found for this theme, which relates to how conflicting information from authorities during a public health emergency leads the public to perceive the information as unreliable [26], contributing to a decline in trust [27]. One team of researchers reported on findings representing the general public and vulnerable populations, indicating:

Participants felt that they had been given contradictory information about when to stay home and when to go to work or school which left them feeling uncertain 


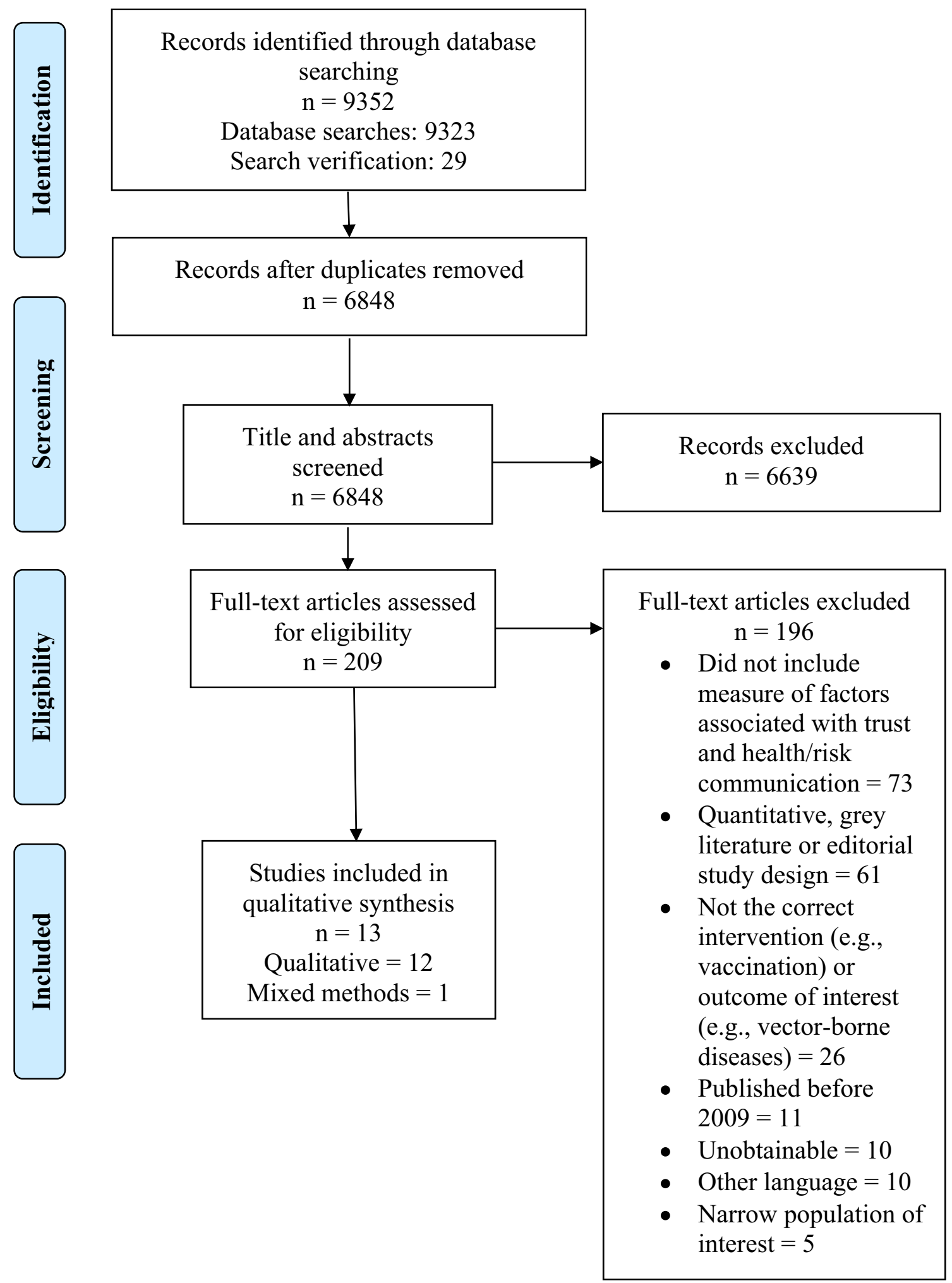

Fig. 1 Flow diagram depicting the flow of information through the different phases of the systematic review, adapted from Moher et al., 2019

about what to do. Consistency of advice is a significant important factor in communications from key agencies. [27], para. 77)
Conflicting information results in the public not trusting that stakeholders were sharing all the information around 
Table 1 Summary

characteristics of the relevant

(13) qualitative studies included in this review examining risk communication and trust

\begin{tabular}{|c|c|c|}
\hline Characteristic & $\mathrm{N}$ & $\%$ \\
\hline \multicolumn{3}{|l|}{ Document type } \\
\hline Journal article & 12 & 92 \\
\hline Dissertation & 1 & 8 \\
\hline \multicolumn{3}{|l|}{ Study Country ${ }^{a}$} \\
\hline Canada & 5 & 62 \\
\hline USA & 3 & 23 \\
\hline Finland & 1 & 8 \\
\hline Australia & 1 & 8 \\
\hline Taiwan & 1 & 8 \\
\hline New Zealand & 1 & 8 \\
\hline Singapore & 1 & 8 \\
\hline Israel & 1 & 8 \\
\hline \multicolumn{3}{|l|}{ Qualitative data collection methods ${ }^{a}$} \\
\hline Interviews & 8 & 62 \\
\hline Content analysis & 6 & 46 \\
\hline Focus groups & 3 & 23 \\
\hline \multicolumn{3}{|l|}{ Infectious disease focus } \\
\hline Pandemic H1N1 & 5 & 38 \\
\hline COVID-19 & 2 & 15 \\
\hline SARS & 1 & 8 \\
\hline Ebola & 1 & 8 \\
\hline Fictious EID & 1 & 8 \\
\hline Unspecified EID & 3 & 23 \\
\hline \multicolumn{3}{|l|}{ Participant type included ${ }^{a}$} \\
\hline Public health officials & 6 & 46 \\
\hline Journalists & 5 & 38 \\
\hline Healthcare workers & 3 & 23 \\
\hline General public or segment of the general public & 3 & 23 \\
\hline Scientists & 2 & 15 \\
\hline Public relations professionals & 2 & 15 \\
\hline Policy makers & 2 & 15 \\
\hline Health bloggers & 1 & 8 \\
\hline \multicolumn{3}{|l|}{ Quality assessment met by each article (yes vs no) } \\
\hline $\begin{array}{l}\text { Research design and data collection strategy clearly described and appropriate to address } \\
\text { the research aims }\end{array}$ & 13 & 100 \\
\hline Sampling strategy clearly described and appropriate to address the research aims & 13 & 100 \\
\hline Method of analysis clearly described and appropriate to address the research aims & 12 & 92 \\
\hline Findings clearly described and supported by sufficient evidence & 13 & 100 \\
\hline Evidence of researcher reflexivity & 9 & 69 \\
\hline Ethical issues taken into consideration & 10 & 77 \\
\hline Evidence of study relevance and transferability & 13 & 100 \\
\hline
\end{tabular}

${ }^{a}$ Multiple selections were possible for these questions, so answers may not add to $100 \%$ a situation, which impacted their ability to make informed decisions [27].

\section{Repetition of the Message}

Moderate confidence was found for this theme, relating to the repetition of key messages surrounding uncertainty to build and maintain public trust and authority [28]. Lohivina et al. [26] conducted a content analysis of social media messages and emails from the public during early COVID-19 in Finland found that repeating risk protective measures and reasoning behind information was important for maintaining trust in authorities "Risk communication recommendations included repeating and explaining information given earlier 


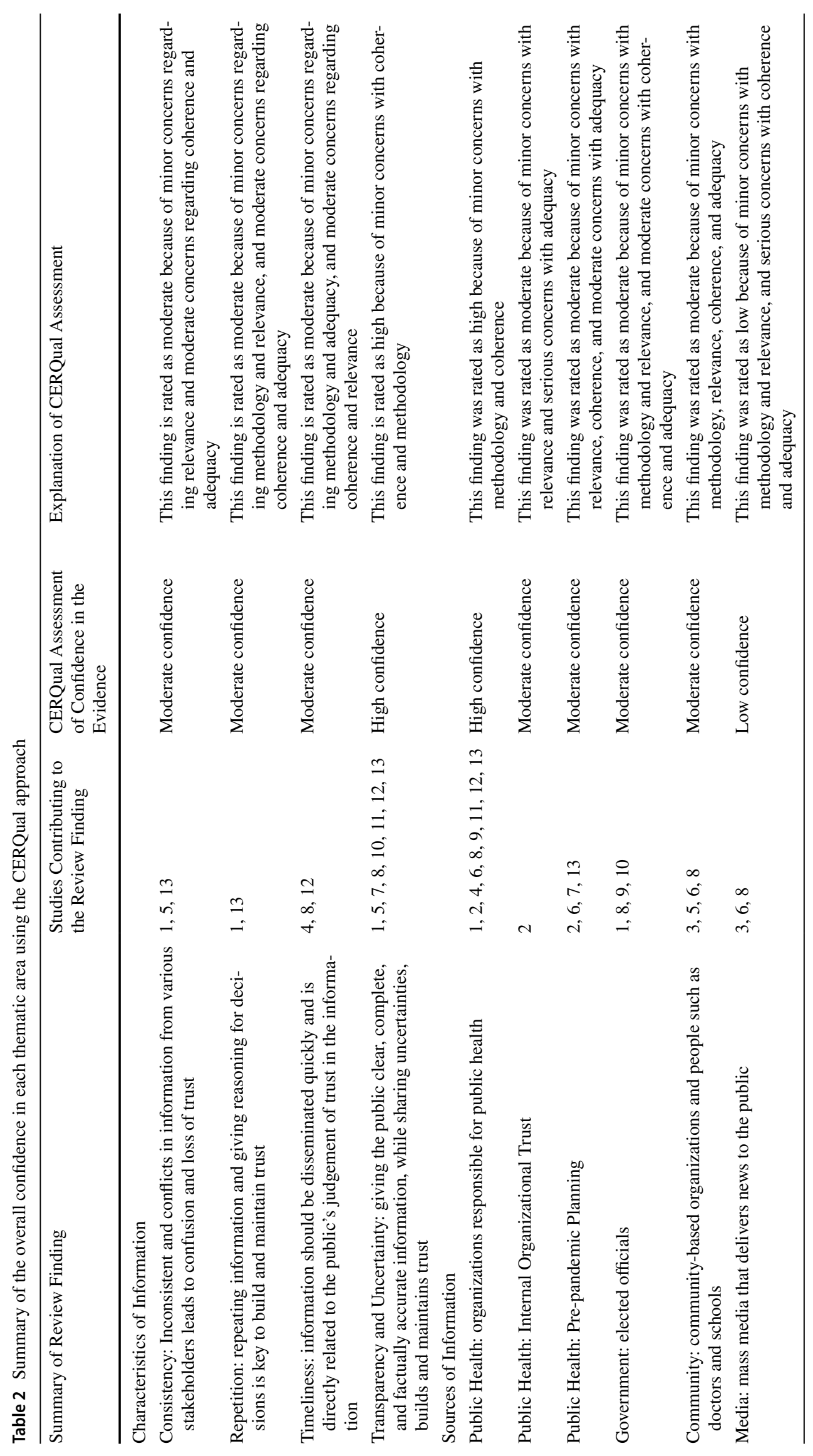




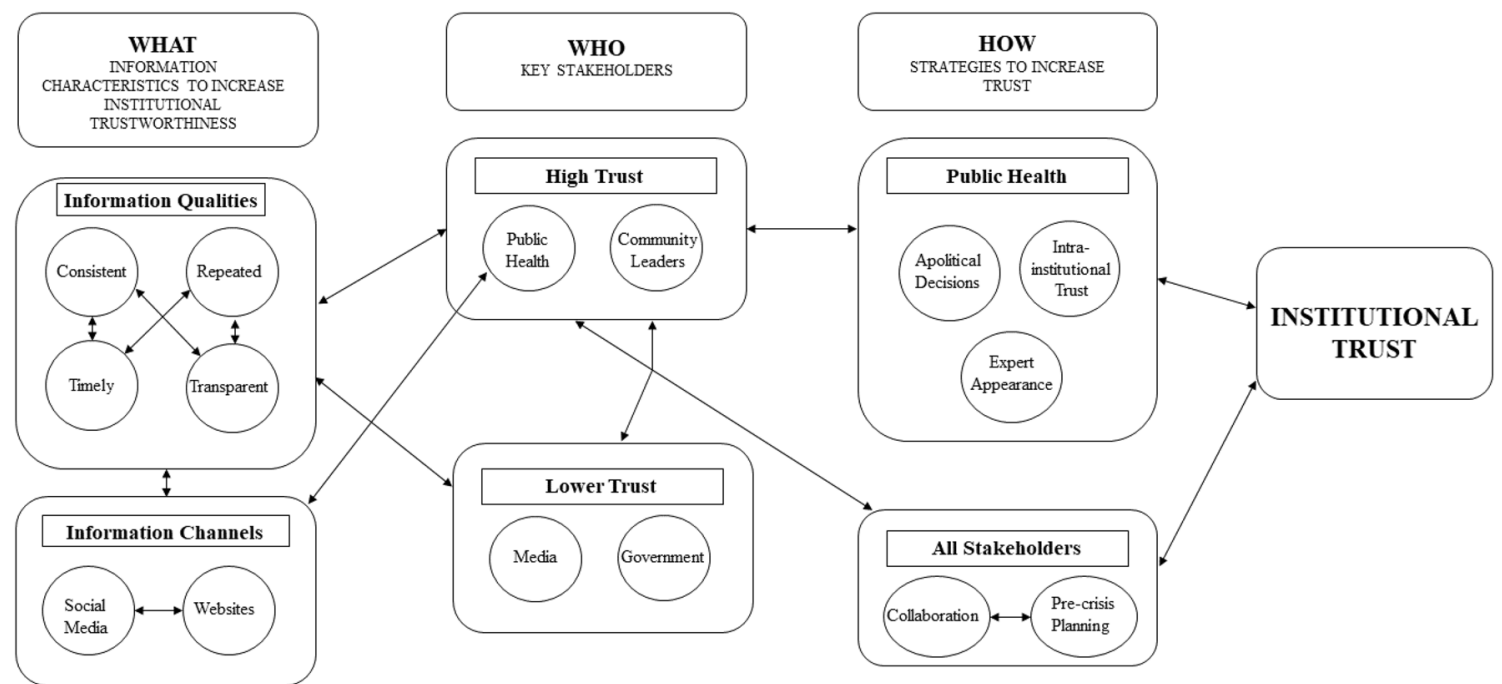

Fig. 2 Conceptual Framework of Essential Factors for Effective Communication Impacting Trust in Crisis Communication

to the public, and communicating the actions taken" ([26], para. 10).

\section{Timeliness of the Information}

Moderate confidence was found for this theme, describing the need for risk and crisis communication to be timely. A public health information officer noted the importance:

I always think of being first, being right, and being credible. So, getting information out as quickly as we can and being as accurate as we can, providing updates when we don't know everything at first and if we find out more info. [29], p. 172)

It is important for public health to disseminate information quickly and to the right audiences, as the public heavily judges a source's trustworthiness by its timeliness [30].

\section{Transparency and Uncertainty when Delivering the Message}

Transparency was a key factor in maintaining public trust [27, 30-32], with high confidence found for this theme. Transparency was associated with clear, complete, and factually accurate information [30]. If the public perceives they are not being given all the facts, it negatively impacts their trust [27]. The importance of transparency is highlighted in the CDC and WHO risk and crisis communication guidelines and reflected by the authors: "Transparency characterizes the relationship between the outbreak managers and the public. It allows the public access to the information-gathering, risk-assessing and decision-making processes associated with outbreak control" [30].
Also impacting the public's trust, and an important aspect of transparency, is the acknowledgement of uncertainty through open and honest communication about what is known and unknown [27, 30]. The unwillingness to communicate uncertainty to the public causes people to perceive messages as unreliable [26], and causes distrust and even panic $[31,33]$. A public health official in one study stated:

You have to be as certain as you can be and even if you need to share it and you're not certain you've got to convey that uncertainty. I think conveying uncertainty is more reassuring than conveying certainty and being proven wrong very soon thereafter because that is once again about trust. [31], p. 799)

\section{Importance of Public Health Agencies as the Messenger}

High confidence was reported for this theme as public health was a key actor during EID. To plan and implement appropriate crisis communication, public health must work quickly to gather information, identify the right spokesperson(s), and collaborate with other key stakeholders [29]. Trusted spokespersons were key to adding credibility and should be clearly identified as a recognizable health professional to increase trust [27, 34] and should work across cultures and political systems [30]. Public health measures, such as travel restrictions and isolation, also must be implemented rapidly by public health or else information and recommendations may be perceived as unreliable, negatively impacting trust [26].

During the 2014-2015 Ebola virus outbreak, a lack of trust in the CDC may have been related to the public's 
perception that the CDC lacked preparedness, expertise, and experience concerning Ebola and that the response was politicized rather than based on scientific evidence [33].

Public health should also use websites and social media to communicate crisis information. Websites give people control over the information they receive and are perceived as less biased and more reliable than traditional media [4]. Social media can be used to engage with communities to build persuasive messages around EID by communicating organization competency and the efficacy of recommendations, reducing uncertainty and building trust through twoway communication [33, 35]. A diverse group of public health professionals illustrates how social media can expand to reach certain populations:

[Using] social media to reach groups we know use it as well. Youth, for example, or in the context of sexual health messaging perhaps through things like Tinder. It's not just a matter of who can't you reach but is there anyone new you can reach through a lot of these tools, as they develop and evolve. [35], p. 862)

\section{United Public Health Voice}

Moderate confidence was found for this theme which describes the need for intraorganizational trust within public health. The approval process for social media messaging within public health can impact the timeliness of messaging. Slow approvals mean conversations will proceed without a public health presence, which has a significant impact on whether the public will act on recommendations and the credibility of public health [35].

Getting through all of the approvals not just from executive approvals at the top. But, also through the program team approvals, having to get buy-in from everybody about the kind of messaging that was going out. That impacted your timeliness in that, as well, because as you go through the approvals process, sometimes the conversation has already occurred, and you're no longer part of it. [35], p. 863)

\section{Public Health Should Establish Positive Relationships and Build Skills Before a Pandemic}

Moderate confidence was found for this theme, describing how pre-pandemic planning can help establish or enhance trust. Luth et al. [34] suggest that public health partner with news media for effective crisis communication, as media amplifies messages while public health adds credibility [28]. The media reports trusting public health spokespersons when they directly participate in decision-making regarding the infectious disease response [36] and spokespersons are doctors as they are perceived to provide more impartial information [28].

Training public health officials and the media to effectively communicate during a crisis was identified as important to maintaining credibility and an effective response [28]. One medical officer of health interviewed stated:

[T]raining to speak to the media in public health should be a top priority... I didn't have any training and I got it on the job... if you're not an effective communicator as a public health official, it affects your credibility and your effectiveness... [28], p. 147)

Media misrepresentation diminished public trust and credibility during the H1N1 pandemic through emphasizing public dissatisfaction in the response to $\mathrm{H} 1 \mathrm{~N} 1$, despite the limited number of cases [28]. A public health information officer noted the importance of effective collaboration in this regard:

We have to make sure we have good relationships with media partners because if you don't, it can affect how our messages are perceived by the public because it's all about how they edit their story, the tone they use, and how they frame the story to the public. [29], p. 172)

Trust can also be built with the public through involving them in discourse surrounding emerging infectious disease planning $[4,31]$. Establishing trust prior to an emergency is important so that when an incident occurs, the public is more likely to view public health as credible, reliable, and trustworthy [35].

\section{Perception of Government}

Moderate confidence was found for this theme, describing public trust in the government and factors impacting that trust. One public health professional said: "I think there's so much skepticism. People don't trust the government as much as they used to and that makes our job difficult" [29], p. 171).

Links to political parties and politicians were associated with distrust in crisis communication [29], while defensiveness in communications by government officials [34], unreliable information, and insufficient restrictions during a pandemic further eroded trust [26]. Collaboration with other credible sources, like medical and health professionals, contributes to the amplification and dissemination of messaging despite declining trust in government [29]. Communicating how necessary the actions of individuals are during EID is essential to compliance with risk protective measures so that the public understands their role in the government response [32]. Finally, speaking directly to the public in their native language, without the 
use of a translator, is also seen as crucial to building trust in government [32].

\section{Perception of Community Leaders}

Moderate confidence was found for this theme, describing trust in community leaders and the need for public health to collaborate with them. Community leaders, including family doctors, childcare staff, and hospital staff, were seen as highly credible sources of information and preferred spokespersons [27]. Staff from hospitals, schools, and childcare centres are seen to be knowledgeable about local circulating virus strains during pandemic influenza, which leads to trust [14]. Public health communicators indicated the importance of working with trusted community organizations:

[They] have been around for years and really work with the community talking about the resources that the government has funded and have worked to disseminate a lot of that information [29], p. 173).

Family physicians were viewed as knowledgeable and trustworthy [14], with trust resulting from the personal patient-physician relationship [4]. This trusting relationship was seen across different groups including university students, adults, new immigrants, parents, and healthcare workers [4].

\section{Perception of Media}

Low confidence was found for this theme, which examines factors associated with trust in the media. Mainstream media (i.e., newspapers and television news) raise awareness of health crises and is an important first source for many [4]. However, after learning about crises from the media, people frequently sought out more credible sources of information as traditional media was often perceived as highly untrustworthy [4]. Several participants including medical officers of health and public relations practitioners indicated: "[T] he media tend to have a distorted view of what is important because their view is colored by what's going-the language is sell more newspapers, right" [31], p. 800). For the public to view information from the media as accurate and complete, information reported in the media should come directly from senior health officials [4].

\section{Discussion}

This review synthesized the findings of primary qualitative literature exploring the factors associated with maintaining trust in key stakeholders' crisis communication during EID in high-income countries. This research builds on previous research that has systematically assessed the literature associated with effective crisis communication during public health crises [37, 38]. The review by Siegrist and Zingg [38], identified that the actions needed for the public to trust, accept, and implement public health recommendations was a gap in the research. The novelty of our research is the focus on qualitative research to understand how trust is built and maintained during EID through factors associated with effective crisis communication that positively influence trustworthiness and the adoption of risk protective measures. Key actors including public health, news media, government, and the public have contributed perspectives on factors associated with trust in crisis communication in the current research. Interestingly, the factors associated with trust in crisis communication found in this research overlap with the conceptual framework developed by Seeger et al. [37] and the existing guides and frameworks surrounding the topic $[5,8,39,40]$. We note that characteristics of crisis communication including transparency, consistency, repetition, and timeliness can demonstrate the trustworthiness of an institution. Furthermore, trust in key stakeholders results from the positive beliefs about the attitudes and actions of sources, which can be enhanced through collaboration, precrisis planning, and choosing the right spokesperson. These factors were also deemed to be crucial elements of successful message development and dissemination, which were found to impact the reach and acceptance of crisis messages, source credibility, understanding, risk perception, and ultimately uptake of public health measures [37, 40].

Trustworthiness is a characteristic of a trusted organization and is influenced directly by the strategies and actions of said organization [41]. The public's perception of institutional trustworthiness and credibility is a significant predictor of the success of public health's actions [13]. Although often used interchangeably, trustworthiness is an attribute of an organization, whereas trust is a belief about the attitudes and behaviours of an organization and is mediated by trustworthiness [41]. Communication contributes to trustworthiness due to its critical role in relationships [41]. Trust can be developed by focusing on building trustworthiness through focusing on the drivers, including effective communication $[37,41]$. Transparency, timeliness, acknowledging uncertainty, and the use of multiple channels have all been highlighted as trust-building features of risk and crisis communication $[5,8,37,39$, 40], through demonstrating organizational trustworthiness. 
High confidence was found for transparency and uncertainty as critical factors of effective risk and crisis communication to build and maintain trust via demonstrating trustworthiness. Transparency was ranked as the most important strategy in crisis communication to maintain trust $[8,42]$. Transparent communication is candid, clear, and accurate [8, 15, 27, 29, 30, 37, 40, 43]. It involves being truthful and showing evidence behind decisions, avoiding over or underestimating threats, acknowledging uncertainty $[8,12$, 40] and takes accountability for when things go wrong [ 42 , 44]. Acknowledging what is unknown is also a key aspect that helps the public understand why new information occurs regularly and why recommendations also change $[8,38,42$, 45]. Providing transparent information increases the likelihood the public will follow risk protective measures due to increased understanding of the situation $[8,37,42,44$, 45]. Transparency is also a key aspect of influencing vaccine uptake and overcoming vaccine hesitancy [46].

Other characteristics that lead to building and maintaining trust through enhancing trustworthiness included consistency, repetition, and timeliness, with moderate confidence found in these themes. Consistency ensures risk and crisis information from within and between organizations is not conflicting [26-28, 37], and empowers the public to engage in recommended protective measures [15, 37, 43]. Repetition involves emphasizing key messages focused on recommended behaviors, decision-making, and uncertainty, and repeating information backed by clear evidence to demonstrate confidence in risk management $[8,15,26,28]$. Finally, timeliness helps maintain trust through quick information dissemination to the right audiences [8, 29, 30, 36, 37, 43]. Although timeliness was not defined in the articles pertaining to this theme, the first source of communication is often more trusted and becomes the benchmark for future communication [44]. Timeliness is more important than crafting a complete message, and a delay in sharing information leads to the perception of lack of transparency and impacts how quicky risk protective measures are accepted [37].

High confidence was found in public health as trusted source of crisis communication when several factors are present. Public health is trusted source of action and information during the COVID-19 pandemic in Canada [47]. Targeting and tailoring information and strategies during pandemics is crucial to ensure information is understood and able to be applied $[8,37]$ and is an important strategy to help overcome the disproportionate impact EID have on racialized communities and other priority populations $[8$, 45]. Social media is an important communication channel $[4,33]$, and posts are more likely to be trusted and engaged with when shared by a public figure already trusted [11]. Two-way communication with the public using social media and websites can better include societal values, through discourse to better understand community information needs, and help demonstrate trustworthiness and maintain trust [4, $14,40,48]$.

Other sources of risk and crisis information included media, community, and government, with low confidence found for media and moderate confidence found for community and government as trusted sources. Media is an important [4], yet mistrusted source of information during COVID-19 [49]. Media use, including newspaper and radio, during COVID-19, is associated with lower knowledge of COVID-19 and prejudice [50]. Public health messaging should be politically neutral, provide targeted and tailored messaging, and address misinformation to counter the negative effect of some news media consumption [45, 50]. While collaboration with the government is essential for public health during an EID, public health must maintain autonomy through transparent communication about the evidence, why decisions have been made or altered, and their support for risk management that differs from that of government's when necessary [44]. To enhance trust in risk and crisis communication at the local level, public health should consider collaborating with community-based organizations to appoint local spokespersons from hospitals, family health teams, childcare centres, and schools [29, 37, 43], as community leaders are seen as trusted sources of information $[4,14,15,27,43]$.

These factors are crucial elements to demonstrating trustworthiness and interact in complex ways that have a positive impact on multiple outcomes. Seeger et al. [37] found that accurate, transparent, and consistent messages contribute to source credibility and trustworthiness, which was also found in this review. Moreover, this research also identified collaboration and the timely dissemination of crisis messages contributes to increased understanding, which also influences credibility and trustworthiness [37]. This review confirms that the ultimate goal of the adoption of recommended or mandated public health actions is influenced by the complex and independent relationship of these factors that influence trust $[37,38,40]$.

\section{Limitations}

The findings of this systematic review are somewhat limited by the characteristics of the included studies. Only thirteen studies were qualitative or mixed methods from the original 6848 , which provides a small subset of studies. This may be in part due to the inclusion criteria but also a reflection of the limited qualitative work in this area. Moreover, most studies that included participants focused on those within public health, government, and media responsible for risk and crisis communication, rather than the public. Only three articles included members from the general population, indicating the need for qualitative research on the public's perspective on how to maintain trust. Diversity of participants was also 
very limited in the included studies: only two of the included studies reported including participants from racialized and public health priority groups.

Several limitations exist in the review process. Language bias may be present as only English language articles were included, limiting articles from other non-English speaking high-income countries. The search strategy may also have missed relevant articles; however, it was minimized through pre-testing our search, including multiple databases, hand searching relevant journals, and performing searches for grey literature.

\section{Future Directions}

Research should focus on the public's perceptions regarding crisis communication information characteristics, sources, and channels to better understand how trust is impacted and why. Research specific to priority populations is needed to be able to address their specific needs and reduce disproportionate harms experienced during public health emergencies. Continued assessment of public health crisis communication is necessary to understand whether best practices are being implemented by public health and how to improve the effectiveness to maintain trust.

\section{Conclusion}

Trust is influenced by characteristics of risk and crisis communication information and the information source. Findings from this review suggest transparency and communicating uncertainty, consistency between stakeholders, repetition of key messages, and timeliness are key aspects of maintaining trust. Importantly, high confidence was found for transparency and communicating uncertainty as increased credibility and trust results from providing information that is understandable and evidence-based, allowing the public to understand the decision-making process behind actions. Public health is considered a trusted source of information, especially when information was not politicized, and when spokespersons present as health officials rather than politicians. Moreover, careful collaboration with media, government, and community organizations can amplify public health messaging and enhance overall trust in risk and crisis communication messaging.

Supplementary Information The online version contains supplementary material available at https://doi.org/10.1007/s10900-021-01032-w.

Acknowledgements The authors would like to thank the University of Guelph Library staff for assistance with developing the search strategy and Dr. Ian Young, Ryerson University, for assistance in qualitative systematic review methodology.
Author's Contributions All authors contributed to the study conception and design. Material preparation, data collection, and analysis were performed by Melissa MacKay, Taylor Colangeli, and Daniel Gillis. The draft of the manuscript was written by Melissa MacKay and all authors contributed to previous versions. All authors read and approved the final manuscript.

Funding This work was supported by the University of Guelph under the University of Guelph COVID-19 Research Development and Catalyst Fund [Number 054624].

\section{Declarations}

Conflict of interest The authors have no relevant financial or non-financial competing interests to disclose.

Availability of Data and Material Supplementary material available with this publication. The data that support the findings of this study are available from the corresponding author upon request.

\section{References}

1. Infection Prevention and Control Canada. (2021). Coronavirus (COVID-19) SARS-CoV-2. ipac. https://ipac-canada.org/coron avirus-resources.php

2. Davis, M. (2018). Uncertainty and immunity in public communications on pandemics. Pandemics, Publics, and Politics: Staging Responses to Public Health Crises. https://doi.org/10.1007/ 978-981-13-2802-2_3

3. Glik, D. C. (2007). Risk communication for public health emergencies. Annual Review of Public Health, 28(1), 33-54. https:// doi.org/10.1146/annurev.publhealth.28.021406.144123

4. Henrich, N., \& Holmes, B. (2011). Communicating during a pandemic: Information the public wants about the disease and new vaccines and drugs. Health Promotion Practice, 12(4), 610-619. https://doi.org/10.1177/1524839910363536

5. Henry, B on behalf of the Canadian Pandemic Influenza Preparedness Task Group. (2018). Canadian pandemic influenza preparedness: Communications strategy. Canada Communicable Disease Report, 44(5), 106-109. https://doi.org/10.14745/ccdr.v44i05a03

6. Quinn, S. C., Parmer, J., Freimuth, V. S., Hilyard, K. M., Musa, D., \& Kim, K. H. (2013). Exploring Communication, Trust in Government, and vaccination intention later in the 2009 H1N1 pandemic: Results of a National Survey. Biosecurity and Bioterrorism: Biodefense Strategy, Practice, and Science, 11(2), 96-106.

7. Vaughan, E., \& Tinker, T. (2009). Effective health risk communication about pandemic Influenza for vulnerable populations. American Journal of Public Health, 99(Suppl. 2), S324-S333. https://doi.org/10.2105/AJPH.2009.162537

8. World Health Organization. (2017). Recommendations. Communicating risk in public health emergencies: A WHO guideline for emergency risk communication (ERC) policy and practice. World Health Organization. https://www.ncbi.nlm.nih.gov/books/ NBK540733/

9. Manchanda, R. \& Qureshi, C. (2020, December 14). Build trust in vaccines by investing in community workers. The Hill. https:// thehill.com/opinion/healthcare/530096-build-trust-in-vaccinesby-investing-in-community-workers

10. Caulfield, T. (2020, March 20). Now more than ever, we must fight misinformation. Trust in science is essential. Folio. https://www.ualberta.ca/folio/2020/03/comme 
ntary--now-more-than-ever-we-must-fight-misinformationtrust-in-science-is-essential.html

11. van Zoonen, W., \& van der Meer, T. (2015). The importance of source and credibility perception in times of crisis: Crisis communication in a socially mediated era. Journal of Public Relations Research, 27(5), 371-388. https://doi.org/10.1080/ 1062726X.2015.1062382

12. Public Health Ontario. (2016) EOH Fundamentals: Risk Communication. Public Health Ontario. https://www.publichealthont ario.ca/-/media/documents/f/2016/fundamentals-risk-comm. pdf?la $=$ en

13. Latkin, C., Dayton, L., Strickland, J., Colon, B., Rimal, R., \& Boodram, B. (2020). An assessment of the rapid decline of trust in US sources of public information about COVID-19. Journal of Health Communication, 25(10), 764-773. https://doi.org/10. 1080/10810730.2020.1865487

14. King, C. L., Chow, M. Y. K., Wiley, K. E., \& Leask, J. (2018). Much ado about flu: A mixed methods study of parental perceptions, trust and information seeking in a pandemic. Influenza and Other Respiratory Viruses, 12(4), 514-521. https://doi.org/ 10.1111/irv. 12547

15. National Collaborating Centre for Methods and Tools. (2020, October 8). What are best practices for risk communication and strategies to mitigate risk behaviours? National Collaborating Centre for Methods and Tools, McMaster University. https:// www.nccmt.ca/knowledge-repositories/covid-19-rapid-evide nce-service

16. Tong, A., Flemming, K., McInnes, E., Oliver, S., \& Craig, J. (2012). Enhancing transparency in reporting the synthesis of qualitative research: ENTREQ. BMC Medical Research Methodology, 12(1), 181. https://doi.org/10.1186/1471-2288-12-181

17. McHugh, M. L. (2012). Interrater reliability: The kappa statistic. Biochemia Medica, 22(3), 276-282.

18. Ortiz-Ospina, E. (2019, September 18). The rise of social media. Our World in Data. https://ourworldindata.org/ rise-of-social-media

19. World Population Review. (2021). High Income Countries 2021. World Population Review. https://worldpopulationreview.com/ country-rankings/high-income-countries.

20. Thaivalappil, A., Waddell, L., Greig, J., Meldrum, R., \& Young, I. (2018). A systematic review and thematic synthesis of qualitative research studies on factors affecting safe food handling at retail and food service. Food Control, 89, 97-107. https://doi.org/10. 1016/j.foodcont.2018.01.028

21. Critical Appraisal Skills Program. (2018.). CASP Checklist: 10 Questions to help you make sense of Qualitative research. Critical Appraisal Skills Program part of Oxford Centre for Triple Value Healthcare Ltd. https://casp-uk.net/wp-content/uploads/2018/01/ CASP-Qualitative-Checklist-2018.pdf

22. Walsh, D., \& Downe, S. (2006). Appraising the quality of qualitative research. Midwifery, 22(2), 108-119. https://doi.org/10. 1016/j.midw.2005.05.004

23. Thomas, J., \& Harden, A. (2008). Methods for the thematic synthesis of qualitative research in systematic reviews. BMC Medical Research Methodology, 8(1), 45. https://doi.org/10.1186/ 1471-2288-8-45

24. Lewin, S., Bohren, M., Rashidian, A., Munthe-Kaas, H., Glenton, C., Colvin, C. J., Garside, R., Noyes, J., Booth, A., Tunçalp, Ö., Wainwright, M., Flottorp, S., Tucker, J. D., \& Carlsen, B. (2018). Applying GRADE-CERQual to qualitative evidence synthesis findings-paper 2: How to make an overall CERQual assessment of confidence and create a Summary of Qualitative Findings table. Implementation Science, 13(1), 10. https://doi.org/10. 1186/s13012-017-0689-2

25. Lewin, S., Glenton, C., Munthe-Kaas, H., Carlsen, B., Colvin, C. J., Gülmezoglu, M., Noyes, J., Booth, A., Garside, R., \&
Rashidian, A. (2015). Using qualitative evidence in decision making for health and social interventions: An approach to assess confidence in findings from qualitative evidence syntheses (GRADECERQual). PLOS Medicine, 12(10), e1001895. https://doi.org/10. 1371/journal.pmed.1001895

26. Lohiniva, A.-L., Sane, J., Sibenberg, K., Puumalainen, T., \& Salminen, M. (2020). Understanding coronavirus disease (COVID-19) risk perceptions among the public to enhance risk communication efforts: A practical approach for outbreaks, Finland, February 2020. Eurosurveillance. https://doi.org/10.2807/1560-7917.ES. 2020.25.13.2000317

27. Gray, L., MacDonald, C., Mackie, B., Paton, D., Johnston, D., \& Baker, M. G. (2012). Community responses to communication campaigns for influenza A (H1N1): A focus group study. BMC Public Health, 12(1), 205. https://doi.org/10.1186/ 1471-2458-12-205

28. Aylesworth-Spink, S. (2015). Falling in Line: News Media and Public Health Response During the 2009 H1N1 Outbreak in Canada [Doctoral dissertation, Queens University]. QSPACE. https:// qspace.library.queensu.ca/handle/1974/12948

29. Jin, Y., Austin, L., Vijaykumar, S., Jun, H., \& Nowak, G. (2019). Communicating about infectious disease threats: Insights from public health information officers. Public Relations Review, 45(1), 167-177. https://doi.org/10.1016/j.pubrev.2018.12.003

30. Gesser-Edelsburg, A., Mordini, E., James, J. J., Greco, D., \& Green, M. S. (2014). Risk communication recommendations and implementation during emerging infectious diseases: A case study of the $2009 \mathrm{H} 1 \mathrm{~N} 1$ influenza pandemic. Disaster Medicine and Public Health Preparedness, 8(2), 158-169. https://doi.org/10. 1017/dmp.2014.27

31. Holmes, B., Henrich, N., Hancock, S., \& Lestou, V. (2009). Communicating with the public during health crises: Experts' experiences and opinions. Journal of Risk Research, 12(6), 793-807. https://doi.org/10.1080/13669870802648486

32. Wong, C. M. L., \& Jensen, O. (2020). The paradox of trust: Perceived risk and public compliance during the COVID-19 pandemic in Singapore. Journal of Risk Research. https://doi.org/10. 1080/13669877.2020.1756386

33. Dalrymple, K. E., Young, R., \& Tully, M. (2016). "Facts, Not Fear": Negotiating uncertainty on social media during the 2014 Ebola crisis. Science Communication, 38(4), 442-467. https://doi. org/10.1177/1075547016655546

34. Luth, W., Jardine, C., \& Bubela, T. (2013). When pictures waste a thousand words: Analysis of the $2009 \mathrm{H} 1 \mathrm{~N} 1$ pandemic on television news. PLoS ONE, 8(5), e64070. https://doi.org/10.1371/journ al.pone. 0064070

35. Khan, Y., Tracey, S., O'Sullivan, T., Gournis, E., \& Johnson, I. (2019). Retiring the flip phones: Exploring social media use for managing public health incidents. Disaster Medicine and Public Health Preparedness, 13(5-6), 859-867. https://doi.org/10.1017/ dmp.2018.147

36. Lyu, S.-Y., Chen, R.-Y., Wang, S. S., Weng, Y.-L., Peng, E.Y.-C., \& Lee, M.-B. (2013). Perception of spokespersons' performance and characteristics in crisis communication: Experience of the 2003 severe acute respiratory syndrome outbreak in Taiwan. Journal of the Formosan Medical Association, 112(10), 600-607. https://doi.org/10.1016/j.jfma.2012.12.005

37. Seeger, M., Pechta, L., Price, S., Lubell, K., A, D., Sapru, S., Chansky, M., \& Smith, B. (2018). A Conceptual Model for Evaluating Emergency Risk Communication in Public Health. Health Security, 16(3). https://doi.org/10.1089/hs.2018.0020

38. Siegrist, M., \& Zingg, A. (2014). The role of public trust during pandemics. European Psychologist, 19(1), 23-32. https://doi.org/ 10.1027/1016-9040/a000169 
39. Centres for Disease Control. (2018). CERC: Introduction. Centre for Disease Control. https://emergency.cdc.gov/cerc/ppt/CERC_ Introduction.pdf

40. Gesser-Edelsburg, A., Shir-Raz, Y., Walter, N., Mordini, E., Dimitriou, D., James, J., \& Green, M. (2015). The public sphere in emerging infectious disease communication: Recipient or active and vocal partner? Disaster Medicine and Public Health Preparedness, 9(4), 447-458. https://doi.org/10.1017/dmp.2015.31

41. Sekhon, H., Ennew, C., Kharouf, H., \& Devlin, J. (2014). Trustworthiness and trust: Influences and implications. Journal of Marketing Management, 30(3-4), 409-430. https://doi.org/10.1080/ 0267257X.2013.842609

42. Henderson, J., Ward, P. R., Tonkin, E., Meyer, S. B., Pillen, H., McCullum, D., Toson, B., Webb, T., Coveney, J., \& Wilson, A. (2020). Developing and maintaining public trust during and postCOVID-19: Can we apply a model developed for responding to food scares? Frontiers in Public Health, 8, 369. https://doi.org/ 10.3389/fpubh.2020.00369

43. Ghio, D., Lawes-Wickwar, S., Tang, M., Epton, T., Howlett, N., Jenkinson, E., Stanescu, S., Westbrook, J., Kassianos, A., Watson, D., Sutherland, L., Stanulewicz, N., Guest, E., Scanlan, D., Carr, N., Chater, A., Hotham, S., Thorneloe, R., Armitage, C., Arden, M., Hart, J., Byrne-Davis, L., Keyworth, C. (2020, July 13). What influences people's responses to public health messages for managing risks and preventing infectious diseases? A rapid systematic review of the evidence and recommendations. https://doi.org/10. 31234/osf.io/nz7tr

44. Sauer, M., Truelove, S., Gerste, A., \& Limaye, R. (2021). A failure to communicate? How public messaging has strained the COVID19 response in the United States. Health Security. https://doi.org/ $10.1089 / \mathrm{hs} .2020 .0190$
45. Skovdal, M., Pickles, M., Hallett, T. B., Nyamukapa, C., \& Gregson, S. (2020). Complexities to consider when communicating risk of COVID-19. Public Health, 186, 283-285. https://doi.org/ 10.1016/j.puhe.2020.07.015

46. Dubé, E., Gagnon, D., \& Vivion, M. (2020). Optimizing communication material to address vaccine hesitancy. Canada Communicable Disease Report, 46(2-3), 48-52. https://doi.org/10.14745/ ccdr.v46i23a05

47. Waddell, C. (2020, May 22). Carleton Researchers Find Canadians Most Trust Public Health Officials on COVID-19. Carleton Newsroom. https://newsroom.carleton.ca/2020/carleton-researchers-find-canadians-most-trust-public-health-officials-on-covid-19/

48. McFadden, S. M., Malik, A. A., Aguolu, O. G., Willebrand, K. S., \& Omer, S. B. (2020). Perceptions of the adult US population regarding the novel coronavirus outbreak. PLOS ONE. https://doi. org/10.1371/journal.pone.0231808

49. John, M. (2021, January 31). Public Trust Crumbles amid COVID, fake news-survey. Reuters. https://www.reuters.com/article/ health-coronavirus-global-trust-idUSL8N2JM2V9

50. Dhanani, L., \& Franz, B. (2020). The role of news consumption and trust in public health leadership in shaping COVID-19 knowledge and prejudice. Frontiers in Psychology. https://doi.org/10. 3389/fpsyg.2020.560828

Publisher's Note Springer Nature remains neutral with regard to jurisdictional claims in published maps and institutional affiliations. 